\title{
Exemple de muticulturalité chez Paul Claudel : les « autre » croyants de trois ouvres
}

\author{
Guila Clara Kessous \\ Harvard University
}

Le commentaire que fait P. Claudel de la Meguillat Esther (Livre d'Esther), livre biblique au fondement de la fête juive de Pourim est particulièrement significatif de son rapport à l'« autre croyant ». Travaillant sur la traduction latine de la Bible, la Vulgate, P. Claudel commente l'histoire à partir du nom d'Esther " occulta abscondita », « la cachée ». Il précise que Mordechai, l'oncle de la jeune Esther, avait disposé devant sa nièce un miroir. L'auteur alors de commenter : "Esther a vu Esther. Elle se tait. Elle a compris ${ }^{1}$. » Pour lui, cette vision catoptrique renvoie au mythe d'Amaterasu « que les Japonais si souvent se sont plus à représenter sur leurs thêâtres ${ }^{2}$ ». L'iniquité des hommes a forcé le Soleil à se cacher « au fond de l'abîme ${ }^{3}$ ». Les dieux ${ }^{4}$, selon P. Claudel, pour l'en faire sortir, font appel à une femme qu'ils placent « au seuil de la caverne ${ }^{5}$ ». Celle-ci, une fois seule, se met à danser. Le Soleil, mécontent du bruit des chants et de la danse, « entr'ouvre la porte, et son premier rayon, son premier regard rencontre ce visage qui n'est autre qu'un miroir. Il s'avance, fasciné, et la porte se referme derrière lui. Elle ne s'ouvrira plus ${ }^{6}$. 》 Amaterasu, 《天照 » en japonais, signifie littéralement « la Soleil », que P. Claudel apparente au personnage d'Esther.

Cette façon de « re-raconter » l'histoire d'Esther est particulièrement intéressante, d'autant qu'il n'existe pas de miroir dans la version biblique originale. Le recours de l'auteur aux différentes cultures (la culture juive travaillant à partir de la source de l'Ancien Testament et la mythologie shintoïste japonaise) va lui permettre, par une opération de syncrétisme, d'en « christianiser » l'histoire. 


\section{Guila Clara Kessous}

Et en commentateur d'Esther, Claudel réécrit la lecture plus que l'Ecriture. Sa polylecture multiplie le divin pour mieux en faire le lien de l'humain au divin, en renforçant la contradiction, même entre le langage désir et l'impossibilité de dire le divin, entre savoir et non-savoir, à travers la Vulgate comme substitut de l'hébreu d'origine, entre le divin biblique et la mythologie, entre la mythologie et le poème?

Nous nous attacherons ainsi tout particulièrement à la « muticulturalité », c'est-à-dire à la juxtaposition de plusieurs imaginaires culturels dans la façon d'envisager le religieux sur scène. Pour ce faire, nous nous pencherons sur la représentation de l'« autre " croyant dans Tête d'Or, Le Soulier de Satin et cette œuvre méconnue qu'est On répète Tête d'Or, réécriture du drame par l'auteur en 1949. Nous employons un caractère minuscule pour désigner l'« autre » en tant qu'image, double spéculaire ${ }^{8}$ en négatif duquel s'établit la propre croyance de l'auteur. La confrontation avec la culture de l'« autre » croyant aboutit sur scène à un théâtre de profonde crise spirituelle. Il s'agit d'une lutte métaphorique de la « lumière » catholique qui tente de se frayer un chemin au travers des «ténèbres » des autres croyances. Simon Agnel et Rodrigue cherchent tous deux à faire briller ce soleil de la foi catholique, même si l'on peut distinguer, dans ces pièces, une profonde évolution du rapport à l'« autre » du point de vue scénique au travers des soixante ans qui séparent Tête d'Or et On répète Tête d'Or. L' « autre » croyant passe de l'ennemi clair, identifiable, dans Tête d'Or à cet « autre » mystérieux, insaisissable, caché « derrière le rideau $^{9} »$ et qui ne se laisse plus appréhender aussi facilement dans son altérité, causant l'interruption de l'écriture dramatique. 


\section{Tête d'Or : l'« autre » croyant face à Simon Agnel}

Le drame Tête d'Or est l'avènement du règne solaire de Simon Agnel, premier " conquistador » pourrait-on dire, venu annoncer un temps glorieux de conquête absolue auquel ne résiste aucun pouvoir de contestation. La réalisation pour le héros, d'avoir été un usurpateur, un faux Messie, ( Je ne suis pas un dieu!10"), d'avoir cru un instant pouvoir se substituer à l'« Autre », sera l'aveu de l'échec d'une possibilité de survie identitaire sans recours à l'« autre ». En effet, Simon Agnel annule l'« autre » croyant en détruisant ce miroir identitaire d'une façon radicale et violente.

LE ROI : Ne prends point ce qui est à moi ; ne dépouille pas ma fille. Car où sera la bénédiction qu'il y aura entre les hommes,

Si tu foules aux pieds la loi sacrée de l'héritage? TETE D'OR : [...] Ton droit, je ne sais pas ce que c'est. Mais pour moi, rejeté de tous, j'ai juré dans mon malheur et dans ma solitude,

Par l'air, par la terre,

Que je m'élèverais au-dessus de la volonté des autres. [...]

J'entre ici et je réclame le Livre et la Couronne!

Retire-toi de devant moi, Vieillard!

LE ROI : Je ne te laisserai point passer.

TETE D'OR : Retire-toi de devant moi, Vieillard! Car ton temps est fini et la nuit est passée, après laquelle un autre jour commence. [...]

LE ROI : Je ne te laisserai point passer.

TETE D'OR : Ne veux-tu point te retirer?

(Le Roi secoue la tête)

Meurs donc!

Il tire son épée et le tue.

Frémissement et confusion dans la foule [...] Les assistants des premiers rangs sont extrêmement pâles et se tiennent comme fascinés, regardant le sang qui se répand 


\section{Guila Clara Kessous}

largement sur le parquet, avec une expression d'horreur et de curiosité. Tête d'Or rit ${ }^{11}$.

L'extrême violence du meurtre est d'autant plus saisissante de par la rapidité de l'action et le refus d'un recours quelconque à une réaction émotive. Ce «meurs donc!» de Tête d'Or peut ainsi être interprété d'une façon héroïque, renvoyant au commandement de Don Diègue au Cid : "Meurs ou tue ${ }^{12}$ ». Il s'agit de l'honneur de Simon Agnel qui se définit comme "l'homme nouveau » venu remplacer l'« homme du temps présent » qui « est déjà passé $^{13}$ ». Le temps du règne de l' " autre » est ainsi passé et avec lui cette « loi de l'héritage » que Simon Agnel refuse.

Le ROI : Et moi, David, le roi aux cheveux blancs, [...]

Je retiens avec mes mains mon âme qui saute !14

Ce Roi David peut renvoyer au souverain du peuple d'Israël (1004-965 avant l'ère chrétienne) qui fit de Jérusalem sa capitale. Il est l'emblème de cette tradition patriarcale puisqu'il lègue le royaume à son fils Salomon qui édifiera le temple. A la verticalité de l'ordre de pensée de l'« autre » croyant, du Roi, Tête d'Or va substituer une ère de l'horizontalité. Rompant le déroulement vertical, diachronique, qui traverse les générations, dans une démarche de " transmission ${ }^{15}$ » à travers le temps, Simon Agnel raie, barre, nie horizontalement ce temps du passé pour le faire basculer dans un registre de "communication » prenant en compte l'espace "géographique " (il se bat avant tout pour la conquête de la « Terre $\left.{ }^{16} »\right)$. Cette « communication » que Tête d'Or est venue donner à l'« autre » est, bien sûr, liée au message divin dont le héros a été investi (épisode de la révélation sous l'Arbre et du cérémonial de baptême de Cébès par le sang dans la première partie). Tête d'Or interrompt la filiation du règne de l'« autre » pour y substituer la « bonne nouvelle » de l'Evangile qu'est l'affiliation, le rattachement à la communauté catholique.

Mais une ère de la communication de la « bonne nouvelle" par le meurtre de l'«autre », en usant de la force pour nier toute 


\section{Exemple de muticulturalité chez Paul Claudel}

existence de l'« autre " ne prend pas en compte la communion des Saints. En effet, cette « communion » désigne la solidarité intime de la communauté terrestre et de la communauté céleste. L' « autre » dans sa croyance, "brebis égarée », doit être sauvé car son âme est liée au salut du peuple catholique dans son entier ${ }^{17}$. Le dernier geste de Tête d'Or, lorsqu'il défait les clous auxquels les mains de la Princesse sont attachées, renvoie à une soudaine prise de conscience de l'« autre » comme étant lié au destin du «Soi ». La générosité de Tête d'Or, qui souhaite rétablir la Princesse sur son trône ${ }^{18}$, rééquilibre les deux axes horizontal et vertical, de la transmission et de la communication, pour les faire se toucher en un point de rencontre, en un baiser, suivant la forme de la croix.

Tête d'Or, rappelons-le, est considéré comme le premier drame de P. Claudel. L'« autre " apparaît donc sous la forme de deux modalités extrêmement simplifiées : soit il "s'ennuie ${ }^{19}$ " et est prêt à croire - c'est le cas de Cébès -, soit il est déjà établi dans sa croyance et en refuse toute autre - c'est le cas du Roi -. Si le premier meurt d'amour à cause de sa croyance, le second, l'ennemi, est anéanti. C'est parce qu'il devait y avoir une autre solution, une autre façon d'envisager l'« autre », en le « convertissant » dans son sens étymologique latin « cum-vertere » signifiant « tourner avec ». La fin de Tête d'Or est donc cette révélation que l'« autre » doit être pris en compte pour converger ensemble vers le divin car toute entreprise solitaire est vouée à l'échec. Lorsque la Princesse «baise sur les lèvres ${ }^{20}$ » Tête d'Or, elle fait acte d'allégeance, acceptant de se «tourner avec » lui vers ce nouvel amour :

\section{LA PRINCESSE : Elle ne t'abandonnera pas, celle que tu as délivrée}

De ta bouche dans le milieu de ses mains sanglantes!

Voilà que tu as délivré celle qui est plus forte que toi!21

Cette Princesse, cette « autre » que Tête d'Or a délivrée est tout à la fois l'emblème de l'âme humaine (Anima), de l'Eglise, de la Vierge et de la Sagesse divine ${ }^{22}$. Mais cette récompense ultime, le couronnement de cette Eglise du dernier acte, ne se fait qu'au 


\section{Guila Clara Kessous}

travers d'un acte d'extrême générosité, d'oubli de soi - au point d'en mourir - de Tête d'Or qui renonce à toute possession matérielle en couronnant l'« autre ».

\section{Le Soulier de Satin: l'« autre » croyant face à Rodrigue}

La «multiculturalité » est, pourrait-on dire, cette toile de fond sur laquelle évolue la fresque historique du Soulier de Satin. Nous pouvons souligner le côté «monstrueux " de l'œuvre : une intrigue comportant quatre-vingts personnages différents, se déroulant sur une vingtaine d'années (en incluant la Quatrième Journée) et dont la durée de représentation avoisine la dizaine d'heures. Rodrigue, au cœur de cette scène qui représente le monde, va aller à la rencontre de l' « autre », s'étant engagé « sous l'étendard qui porte [Le] Monogramme ${ }^{2} »$ divin. C'est donc sous le signe du chrisme $(\mathbb{R})$ que Rodrigue fait sa première apparition " en absence » par le discours initial du Père Jésuite qui explique que Rodrigue est de "ceux-là qui ne peuvent se sauver qu'en sauvant toute cette masse qui prend leur forme derrière eux ${ }^{24}$ ». Cette masse renvoie explicitement aux « autres », ces « autres » croyants et mécréants dans Le Soulier de Satin dont il faut « exprimer », c'est-à-dire "faire sortir en exerçant une pression » un Credo. Or, le Père Jésuite précise dès l'incipit que Rodrigue, tout comme Tête d'Or, souffre de cette envie de " conquérir et posséder $^{25}$ ». Toute l'histoire est donc celle d'un refus de conquête et de possession de l'autre pour aller vers le renoncement, et par là atteindre et faire atteindre la transcendance à l' « autre ». La réalisation, pour Tête d'Or, de l'existence de l'« autre » était insupportable et provoquait la mort. Rodrigue vivra différentes étapes au cours de ces quatre journées pour résister et survivre à son désir de conquête et de possession de l'« autre » pour passer graduellement de l'avoir à l'être, finissant tout comme le Père Jésuite, consentant à suivre la Mère glaneuse ${ }^{26}$. Le périple de Rodrigue visà-vis de l'« autre » est donc le même que celui de Tête d'Or, sauf 
que trente cinq ans plus tard, P. Claudel fait survivre son héros à la révélation de l'altérité.

Le périple de Rodrigue face à l'« autre » croyant commence dès sa première apparition scénique dans le désert de Castille à la scène VII de la Première Journée. D'emblée, Rodrigue est présenté comme lié à un " autre ", Isidore le Chinois, double fantasque.

LE CHINOIS : Oh! ne craignez pas que je fuie, Pour autant que vous respecterez notre pacte et ne me ferez passer la nuit auprès d'aucun cours d'eau, source, puits.

DON RODRIGUE : Crains-tu si fort que je te baptise subrepticement ?27

La peur de cet « autre " est la conversion infligée malgré lui. Ainsi, la première occurrence de Rodrigue dans la pièce est liée au changement de la nature de la croyance de l' " autre » par la force. Mais cette fois-ci, l'« autre »n'est plus ce Roi de Tête d'Or qui se laisse tuer, il résiste.

LE CHINOIS : Et pourquoi vous donnerais-je ainsi pour rien le droit de me faire chrétien et d'entrer au Ciel avec l'ornement que je vous aurai fourni? Et compensation d'autres desseins moins purs,

Il faut d'abord que vous serviez quelque peu Monsieur votre serviteur ${ }^{28}$.

L'« autre » a soudain conscience de la volonté qui lui est imposée et refuse de se "laisser faire », de voir quelqu'un « tripote[r] à leur idée ${ }^{29}$ », non seulement parce qu'il tient à sa nature "croyante », mais aussi parce qu'il réalise les bienfaits spirituels qu'il procure au " convertisseur ". L' « âme en danger ${ }^{30}$ » n'est donc plus si facile à récupérer et va devoir se jouer pour Rodrigue dans une prise en compte de la dépendance à l'« autre » dans son altérité. 


\section{Guila Clara Kessous}

LE CHINOIS : Oui, j'aimerais beaucoup être en d'autres mains que les vôtres, pourquoi m'y suis-je mis?

DON RODRIGUE : C'est moi qui suis dans les tiennes $^{31}$.

Tout le drame se joue ici dans un jeu du «Soi » et du bon vouloir de l'« autre » comme clé de l'« Autre ». Isidore, le Chinois, par exemple, a accepté de se convertir, d'étudier tous les livres à la condition que Rodrigue le mène à la Négresse qui a volé son argent. Don Camille apprend à Doña Prouhèze qu'elle est la clé de son salut et la force à l'épouser à la mort de son mari, malgré sa passion pour Don Rodrigue. Le fils de Doña Musique et du Vice-Roi de Naples est destiné à épouser Sept-Epées, la fille de Don Camille et de Doña Prouhèze, jeune fille au visage de Don Rodrigue. «Ce million de choses qui existent ensemble ${ }^{32}$ » représente cette " masse " que Rodrigue doit sauver.

L'importance de la culture asiatique, ouvrant et clôturant la pièce, est à relever. L'auteur définissait sa pièce sous le signe de l'Asie :

Le sujet du Soulier de Satin, c'est celui de la légende chinoise, des deux amants stellaires qui chaque année après de longues pérégrinations arrivent à s'affronter, sans jamais pouvoir se rejoindre, d'un côté et de l'autre de la voie lactée ${ }^{33}$.

Si Rodrigue apparaît pour la première fois accompagné du Chinois, il débute la Quatrième Journée avec le Japonais, Daibutsu.

Vingt ans se sont écoulés depuis le début de l'œuvre et Rodrigue est à présent « vieilli et grisonnant; il a une jambe de bois $^{34}$ » et se trouve dans une cabine de son bateau. Le Japonais Daibutsu dessine les «Feuilles de Saints », qui sont des illustrations que lui dicte Don Rodrigue : 


\section{Exemple de muticulturalité chez Paul Claudel}

DON RODRIGUE : Achève ton travail pendant que nous sommes chauds tous les deux! Je sens que ça va marcher! Je sens l'inspiration qui me sort jusqu'à l'extrémité de tes dix doigts ${ }^{35}$.

Plus question de conversion ici, mais au contraire une voie d'accès à l'art, au divin par l'« autre ». En effet, Daibutsu, le Japonais, peint à l'écoute des mots de Don Rodrigue, des figures saintes (Vierge et Saints) que Rodrigue décrit comme « ces grandes possibilités de [lui]-même ${ }^{36}$ ». L'« autre » est devenu le moyen ultime d'expression pour atteindre la transcendance, transformant la parole de Rodrigue, le poète, en art. Tout comme l'acteur au théâtre, le peintre use de mots qu'il ne comprend pas (rappelons que le but n'est plus la conversion) pour en faire jaillir une création artistique, en tant qu'intermédiaire nécessaire d'une co-naissance globale. P. Claudel pourrait ainsi parler d'une inspiration qui lui sort de l'extrémité des dix doigts du comédien, qui n'est là que pour « être habité " par la parole de l'auteur et la répandre, la « dire misérablement sur la terre ${ }^{37}$ ». Le rapport à l'« autre » croyant, s'il reste toujours conflictuel, a profondément évolué dans Le Soulier de Satin, devenant une co-naissance qui inclut l'autre dans la destinée individuelle comme nécessaire, non seulement dans une optique sociale, mais également dans une optique religieuse : l'« autre » dans Le Soulier de Satin, devient clé du salut.

\section{On répète Tête d'Or : «Je est un autre »}

Le rapport à l'« autre » croyant est redondant dans l'œuvre dramatique de P. Claudel. Nous avons vu que celui-ci est envisagé comme « intermédiaire » nécessaire, voie d'accès au divin dans Le Soulier de Satin. Cependant, P. Claudel a longtemps rêvé d'ajouter une pièce à la Trilogie des Coûfontaines (L'Otage, Le Pain dur, Le Père humilié) qui aurait mis en scène un dialogue entre le personnage de Pensée, femme juive aveugle (la Synagogue, telle qu'on la retrouve sur les murs de la cathédrale de Strasbourg) avec Sarah, 


\section{Guila Clara Kessous}

fille qu'elle a eue d'Orian, le neveu du pape ${ }^{38}$. Cette pièce revient souvent dans son Journal ${ }^{39}$, sans que l'auteur ne puisse la concrétiser. Cependant, il semble que la pièce inachevée On répète Tête d'Or soit une ultime tentative d'écriture du face-à-face avec l'« autre " croyant, dialogue entre le judaïsme (Pensée) et le christianisme (Sarah, fille de Pensée).

Nous avons souligné dans la première partie l'horreur qu'éprouve $\mathrm{P}$. Claudel devant la possibilité de représenter Tête d'Or, que J.-L. Barrault se propose de mettre en scène :

Ce n'est pas que la pièce manque de sincérité [...]! Mais c'est justement cette sincérité crue, maladroite, horriblement naïve qui me fait frissonner! C'est comme si j'avais à me présenter devant le public, dépouillé non pas seulement de ma chemise, mais de ma peau! A ce point que je ne puis plus lire le livre! [...] Je n'imagine pas la scène de la communion par le sang! [...] Le crucifiement de la "Princesse » (qui est cette Princesse?) et le décrucifiement, la mort dans le soleil comme Hercule sur l'Oeta. A cette pensée un frisson me parcourt l'épine dorsale! On n'a peur de rien quand on a vingt ans ${ }^{40}$ !

Le personnage de Tête d'Or, dans son refus absolu d'envisager l'« autre » dans son altérité, s'élançant à la conquête du monde en tuant l'ordre ancien du Roi David, a des échos bien effrayants après la Seconde Guerre Mondiale. C'est pour cette raison que $\mathrm{P}$. Claudel décide alors de remanier le texte qu'il a écrit soixante ans plus tôt. Il élabore une trame très précise de l'œuvre : le désir de pouvoir de Tête d'Or est mis en relation avec un désir d'infini qui trouve sa forme absolue dans le dialogue avec la Princesse au troisième acte :

Et la voix, cette voix de la Princesse inculpée au IIIème acte du vieux drame, répond:

- Je suis l'Eglise catholique ${ }^{41}$. 
Quant au genre théâtral choisi, l'auteur reprend la forme dramatique qu'il avait utilisée auparavant dans sa pièce Les Fourberies de Scapin, usant d'acteurs qui répètent une pièce qu'ils jouent malgré eux. Pour retrouver le climat de rédaction de 1890, ce « couvercle matérialiste » dont le jeune Claudel souffrait, l'auteur décide de «faire jouer Tête d'Or dans un stalag, par des prisonniers, entre des barbelés et sous le bombardement des avions $^{42} »$.

Si P. Claudel a donc établi des lignes directrices fortes (décor, intrigue, forme dramatique), il lui faut encore approfondir les personnages. C'est ce qu'il choisit de faire comme on l'apprend au travers dans son Journal, au travers de lectures liées à la mystique juive :

Ibn Ezra dans son Livre du Nom dit que le Tout connaît la partie par la voie du Tout et non par la voie de la partie.

Le Sceau de Salomon

$\begin{array}{lll}6 & 7 & 2 \\ 1 & 5 & 9 \\ 8 & 3 & 4\end{array}$

15 dans toutes les directions. Or $15=$ Jh est la moitié du nom de Jéhovah. 5 au centre est le Logos. Les autres chiffres pairs aux quatre angles sont les quatre éléments ${ }^{43}$. Le kabbaliste Nahmanida dit q[ue] Dieu créa d'abord une matière fine, subtile, sans consistance, mais ayant la force potentielle de réceptibilité de la forme, et c'est la matière première hiylé, en grec hylè. De cette matière première Dieu tira [7 V] toutes choses. C'est elle q[ue] la Genèse $(1,2)$ entend par le mot tohu. Puis il revêtit cette matière de forme : c'est le bohu de la Genèse...Il admet q[ue] cette matière a un élément spirituel destiné 


\section{Guila Clara Kessous}

à constituer les esprits et un élément spirituel [sic] destiné à constituer les corps (Serouya, la Kabbale, p. 162$)^{44}$.

Voilà l'auteur se tournant non seulement vers le texte biblique de l'« autre ${ }^{45}$, mais aussi vers un livre ésotérique traitant de l'ensemble des spéculations métaphysiques sur Dieu, l'homme et l'univers, prenant racine dans la tradition mystique du judaïsme. La Kabbale, « קבלה » en hébreu, vient du verbe « recevoir », en relation avec une certaine compréhension du monde en ce sens qu'elle incite à modifier notre perception du monde en ayant recours à des concepts et métaphores-clés de la connaissance humaine, comme l'Arbre de la Vie ou les Sephiroth (formes imagées des forces de la volonté divine). Ce qui est particulièrement intéressant est que P. Claudel a un recours direct à la culture juive dans cette pièce dans la mesure où il donne un nom juif à son héros, qui passe de Simon Agnel à Simon Bar Jona signifiant en hébreu ${ }^{46}$ le « fils de la colombe ». Peu à peu, la pièce s'écrit d'une façon différente de celle que P. Claudel avait imaginée : le tuyau de poële devient l'Arbre sous lequel Simon a sa révélation, mais cet arbre est " le père Abraham ${ }^{47}$ " et s'apparente étrangement à cet Arbre de Vie mentionné dans la tradition juive kabbalistique :

$X$. Alors c'est le tuyau de poële qui va être votre nom de Dieu de bon Dieu et q'vous allez prendre dans vos bras pour vous expliquer le ciel et la terre?

SIMON : Pas seulement le ciel et la terre, mais les dix séphiroth. T'as pas entendu, les dix séphirot?

$\mathrm{X}$. Je sais pas ce que c'est.

SIMON : Moi non plus, mais ça fait bien. J'ai trouvé ça dans un livre: Les dix séphiroth ${ }^{48}$.

Le plus troublant est ce refus de la part du personnage principal de reconnaître l'origine juive, et dans son nom (qu'il dit venir du latin), et dans sa mention des « dix séphirot » (dont il affirme 


\section{Exemple de muticulturalité chez Paul Claudel}

ignorer la signification). Tout se passe comme si P. Claudel luimême résistait à prononcer le mot de cet « autre » embrassant, le « juif » qui va pourtant apparaître, presque malgré l'auteur, sous les traits d'un garçon de café...qui tient le rôle de la Princesse!
SIMON : Qui c'est q'vous avez choisi pour fair' la Princesse pendant que j'étais parti ailleurs autre part pour m'occuper de vous?
$\mathrm{X}$ : C'est c't espèce de youpin, le clairon du $127^{\text {ème. }}$.
$\mathrm{X}:$ C'est pas nous q'la choisi. S'a choisi tout seul ${ }^{49}$.

On peut imaginer l'impasse devant laquelle $P$. Claudel se trouve devant ce garçon de café juif qui représente l'Eglise!

\section{Mais comment s'en tirer??? Vous voyez bien que tout cela est bien difficile ${ }^{50}$ !}

Force est de constater la manière dont la culture de l'« autre » perce et déteint sur l'auteur qui était parti de l'idée de faire de Simon Bar Jona un tuberculeux mourant au crâne rasé, un symbole du Christ. Il semblerait donc que le but de la pièce ait été de montrer le martyr de Tête d'Or qui meurt le jour de Pâques entouré de ses douze apôtres en le faisant dialoguer avec la Princesse, l'Eglise. Cependant, tout change en cours de route car le personnage du garçon de café juif, voix derrière le rideau, s'est choisi lui-même malgré son auteur, pour incarner le rôle de la Princesse...Le fantôme de la Synagogue, cette femme aveugle, Pensée, revient hanter le texte, « horrifiant » l'auteur. Cette voix derrière le rideau se fait entendre, tenant tête à Simon qui peu à peu perd de sa superbe et reconnaît son autorité :

$\mathrm{X}$ : C'est toi la colombe?

SIMON : Oui, ça serait moi, la porte pour vous tous, s'y avait pas ct'aut'fi de la colombe, là-bas derrière le rideau qui s'occupe à me traverser ${ }^{51}$. 


\section{Guila Clara Kessous}

Simon reconnaît être " traversé » par cet « autre » avec lequel il est en concurrence. Fidèle à son personnage de Tête d'Or, il décide de s'en débarrasser en supprimant le rôle :

SIMON : Précisément, je voulais supprimer le rôle de la Princesse.

$\mathrm{X}$ : T'aurais bien fait!

La voix derrière le rideau : Elle est plus forte que toi! [...] $\mathrm{X}$ : Alors, dis-donc, montre nous un petit peu ton museau, qu'on le voie.

La voix : Patience!

SIMON : C'est moi-même, chère Madame, qui me permets de vous le demander.

La voix : Tout à l'heure! Un petit bout de la jeune personne, cher Monsieur, on s'en va vous le montrer tout à l'heure.

Mais sais-tu bien que c'est au troisième acte, seulement toi et moi, que nous aurons notre explication.

Non seulement l'« autre » résiste dans son altérité, mais il se présente également comme le principe même, la source de la divinité, puisqu'il tient lieu d'Eglise pour P. Claudel. L'auteur n'écrit pas cette explication de Simon Bar Jona (le Christ) et du garçon de café (le Judaïsme) du troisième acte. Cela est impossible. Rappelons que dans la pièce originale Tête d'Or, le troisième acte présente un incroyable " coup de théâtre ». La Princesse, chassée par Tête d'Or, sera recouronnée par celui-ci... Ayant souffert de l'errance (Diaspora) et d'injustes souffrances (le Déserteur la crucifie par vengeance de crimes dont elle est innocente), la Princesse retrouve son royaume par celui qui l'avait détrôné...P. Claudel s'arrête, " horrifié » devant cet « autre » qui s'impose à lui dans toute sa gloire, dans une vision dramatique qu'il refuse de faire aboutir : le Judaïsme crucifié recouronné par le Christ...

Le recours à la « multiculturalité » pour P. Claudel aboutit progressivement à une appréhension de l'« autre ", non seulement 


\section{Exemple de muticulturalité chez Paul Claudel}

comme clé du salut, mais aussi comme lieu du divin même dont le «face-à-face » est insoutenable...

\section{Notes}

1 Paul Claudel, Le Poëte et la Bible, tome 1, Gallimard, 1998, p. 465.

2 Paul Claudel, Ibid.

3 Paul Claudel, Ibid.

4 Selon la tradition japonaise, c'est, en fait, son frère Susanoo qui aurait enfermé la jeune fille.

5 Paul Claudel, Ibid.

6 Paul Claudel, Ibid.

7 Henri Meschonnic, « Nous passons en image. Le Champ de figures chez Paul Claudel » dans Jacques Houriez, Claudel, le poëte et la Bible, 2001, Actes du colloque des 16-17 octobre 1998 à la Bibliothèque Nationale de France, $\mathrm{n}^{\circ} 714$, p. 75.

8 Nous nous référons ici aux théories de Jacques Lacan, Le Séminaire livre XVI (1968-1969), D'un Autre à l'autre, éditions du Seuil, Champ Freudien, Paris, 2006.

9 Paul Claudel, On répète Tête d'Or dans Michel Lioure, Tête d'Or de Paul Claudel : introduction, inédits, variantes et notes, Paris, Les Belles Lettres, 1984, p. 296.

10 Paul Claudel, Tête d'Or, Théâtre I, Paris, Gallimard, coll. Bibliothèque de La Pléiade, 1967, p. 281. Dans la suite de cette étude, nous utiliserons l'abréviation TO pour référer à cette œuvre dans l'édition citée précédemment.

11 Paul Claudel, TO, op. cit., p. 242.

12 Pierre Corneille, Le Cid, Euvres complètes, t. 1, Paris, Gallimard, 1980, acte I, scène 5, p. 1435.

13 Paul Claudel, TO, op. cit., p. 241.

14 Paul Claudel, TO, op. cit., p. 193.

15 Nous reprenons ici la distinction transmission/communication de Régis Debray, Transmettre, Paris, Odile Jacob, 1997.

16 SIMON : «Et toi, ô Terre, voici que je m'étends sur ton sein! », Paul Claudel, TO, op. cit., p. 187. 


\section{Guila Clara Kessous}

17 « C'est ainsi que je vous dis qu'il y aura plus de joie dans le ciel pour un seul pécheur qui se repent que pour 99 justes, qui n'ont pas besoin de repentir », Luc 15, 7.

18 TETE D'OR : "Qu'elle soit Reine », Paul Claudel, TO, op. cit., p. 297.

19 Paul Claudel, TO, op. cit., p. 180.

20 Paul Claudel, TO, op. cit., p. 301.

21 Paul Claudel, TO, op. cit., p. 295.

22 Ernest de Beaumont, Le sens de l'amour dans le théâtre de Claudel : le thème de Béatrice, Paris, Lettres modernes, 2000.

23 Paul Claudel, Le Soulier de Satin, Théâtre II, Paris, Gallimard, coll. Bibliothèque de La Pléiade, 1965, p. 668. Dans la suite de cette étude, nous utiliserons l'abréviation $S d S$ pour référer à cette œuvre dans l'édition citée précédemment.

24 Paul Claudel, Ibid.

25 Paul Claudel, Ibid.

26 DON RODRIGUE : « Je veux vivre à l'ombre de la Mère Thérèse! » Paul Claudel, SdS, op. cit., p. 947.

27 Paul Claudel, SdS, op. cit., p. 691.

28 Paul Claudel, SdS, op. cit., p. 692.

29 Ibid.

30 Paul Claudel, SdS, op. cit., p. 693.

31 Paul Claudel, SdS, op. cit., p. 692.

32 Paul Claudel, SdS, op. cit., p. 697.

33 Paul Claudel, Allocution du 23 mars 1944, Théâtre II, Paris, Gallimard, coll. Bibliothèque de La Pléiade, 1965, p. 1476.

34 Paul Claudel, SdS, op. cit., p. 867.

35 Paul Claudel, SdS, op. cit., p. 869.

36 Paul Claudel, SdS, op. cit., p. 871.

37 Paul Claudel, SdS, op. cit., p. 669.

38 Le dialogue d'Orian et de Pensée se retrouve dans la dernière pièce de la Trilogie des Coûfontaines : Le Père humilié. Nous reprenons dans notre développement les analyses de Yehuda Moraly, Claudel metteur en scène, la frontière entre deux mondes, Besançon, Presses Universitaires Franc-Comtoises, 1998, p. 275-314. 


\section{Exemple de muticulturalité chez Paul Claudel}

39 Paul Claudel, Journal I, Paris, Gallimard, coll. Bibliothèque de La Pléiade, 1968, p. 1241.

40 "Lettre de Paul Claudel à Jean Louis Barrault du 6 janvier 1944 ", Cahiers Paul Claudel X, Paris, Gallimard, 1974, p. 134.

41 Paul Claudel, Théâtre I, op. cit., p. 1250.

42 Jean Louis Barrault, Nouvelles réflexions sur le Théâtre, Flammarion, Paris, 1959, p. 250.

43 Paul Claudel va se réapproprier cette numérologie qu'il adaptera au christianisme, faisant du chiffre 13 , somme numérologique des initiales christiques, le nombre symbolique de ses personnages à la fin du drame. Au premier acte, trois prisonniers participent aux répétitions. Au deuxième acte, six personnages anonymes entourent Simon Bar Yona. Si P. Claudel se montre fidèle à cette progression, il s'agirait d'entourer le héros de douze prisonniers, qui tiendrait lieu de douze apôtres puisque la scène finale devrait se dérouler le jour de Pâques.

44 Paul Claudel, Journal II, Paris, Gallimard, coll. Bibliothèque de La Pléiade, 1969, p. 707-708.

45 On sait qu'il était un fervent lecteur de l'Ancien Testament qu'il lisait en latin.

46 Et non pas en latin comme le précise le personnage dans la pièce. L'auteur aurait combiné le nom de l'inventeur de la Kabbale, Rabbi Simon Bar Yochaï, du nom Bar Yohanna, signifiant fils de Jean, renvoyant à l'apôtre Jean l'Evangéliste. Il aurait ainsi trouvé le parfait équilibre entre les deux religions. SIMON : « Bar Jona y paraît q'ça veut dire le fils de la colombe en latin. " Paul Claudel, On répète Tête d'Or dans Michel Lioure, Tête d'Or de Paul Claudel : introduction, inédits, variantes et notes, Paris, Les Belles Lettres, 1984, p. 280.

47 Paul Claudel, Ibid., p. 281.

48 Paul Claudel, Ibid., p. 282.

49 Paul Claudel, Ibid., p. 288.

50 «Lettre de Paul Claudel à Jean Louis Barrault du 2 septembre 1950 », op. cit., p. 219.

51 Paul Claudel, On répète Tête d'Or, op. cit., p. 288. 